Auch das böse Wort vom nach Europa geschickten „Opa“ findet - für die mit dem Ende der 6. Wahlperiode 2009 ausgeschiedenen Europa-Abgeordneten - keine Bestätigung, auch nicht bei jenen Abgeordneten, die nur eine Wahlperiode im EP vertreten waren. Das Europäische Parlament wurde von den Parteien so gut wie gar nicht dazu genutzt, langgedienten Politikern einen parlamentarischen „Alters(ruhe)sitz“ zu gewähren. 54

54 Es kann nicht völlig ausgeschlossen werden, dass es nicht auch eine Art „Gefälligkeitsnominierung“ gab, doch hatte kein Abgeordneter, der nur eine Wahlperiode im EP saß, zum Zeitpunkt des Einzugs in das EP bereits das 65. Lebensjahr erreicht. Gegen eine derartige Vergabe von EuropaMandaten spricht aber auch die ausgeprägte Konkurrenz bei der Nominierung für ein Mandat und auch die Anforderung bei der Listenaufstellung, regionale Parteigliederung und gegebenenfalls auch Partei-Strömungen zu berücksichtigen. Die insgesamt dann doch sehr geringe Zahl von erfolgversprechenden Listenplätzen bietet den Parteien nur in geringem Umfang die Chance für „Gefälligkeitsnominierungen“. Diese Einschätzung wird jedoch nicht dadurch grundsätzlich ins Gegenteil verkehrt, dass für die 7. Wahlperiode Monika Hohlmeier (47, CSU), Reinhard Bütikofer (56, Die Grünen) und Lothar Bisky (67, Die Linke) - Altersangabe jeweils für den Zeitpunkt der Konstituierung des Europäischen Parlaments - erfolgreich nominiert wurden.

\title{
Das novellierte Gesetz über die Zusammenarbeit von Bundesregierung und Deutschem Bundestag in Angelegenheiten der Europäischen Union (EUZBBG)
}

\author{
Hinrich Schröder*
}

Am 13. Juli 2013 trat das neu gefasste Gesetz über die Zusammenarbeit von Bundesregierung und Deutschem Bundestag in Angelegenheiten der Europäischen Union (EUZBBG) ${ }^{1}$ in Kraft. Wie sein Vorgänger ${ }^{2}$ konkretisiert es die in Art. 23 Abs. 2 und 3 des Grundgesetzes (GG) statuierten Unterrichtungs- und Stellungnahmerechte des Bundestages. Es soll einfachgesetzlich sicherstellen, dass der Bundestag von der Bundesregierung über alle Angelegenheiten der Europäischen Union umfassend und zum frühestmöglichen Zeitpunkt unterrichtet wird und sein Recht zur Stellungnahme effektiv wahrnehmen kann.

Eine Änderung des Gesetzes war erforderlich geworden, nachdem das Bundesverfassungsgericht (BVerfG) in seinem Urteil vom 19. Juni $2012^{3}$ die Unterrichtungsrechte des Bundestages nach Art. 23 Abs. 2 GG weiter ausgelegt hatte, als sie vom bisherigen EUZBBG abgebildet waren. Dem Urteil vorausgegangen war eine - nach Inkrafttreten des EUZBBG

* Der Autor ist Referent im Referat EU-Grundsatzangelegenheiten, Fragen der Wirtschafts- und Währungsunion der Verwaltung des Deutschen Bundestages. Der Beitrag gibt ausschließlich seine persönliche Auffassung wieder.

1 BGBl. 2013 I, S. 2170.

2 Vgl. zum EUZBBG a.F. Hinrich Schröder, Mitwirkung des Bundestages in EU-Angelegenheiten nach dem EUZBBG in der Praxis - ein Kurzkommentar, in: ZParl, 43. Jg. (2012), H. 2, S. 250 $-277$.

3 BVerfG, 2 BvE 4/11 vom 19. Juni 2012; BVerfGE 131, S. 152. 
im September 2009 einsetzende - Entwicklung auf EU-Ebene hin zu einer verstärkten intergouvernementalen Zusammenarbeit bei der Bewältigung der Schulden- und Finanzkrise. In diesem Zusammenhang war es zwischen Teilen des Bundestages und der Bundesregierung zu Meinungsverschiedenheiten gekommen, ob auch völkerrechtliche Verträge zwischen Mitgliedstaaten als Angelegenheiten der EU zu behandeln sind und damit die entsprechenden grundgesetzlich verankerten Unterrichtungsrechte auslösen. Das BVerfG beantwortete diese Frage im Sinne des Bundestages. Nunmehr bildet das novellierte EUZBBG die Vorgaben des Urteils ab. ${ }^{4}$ Daneben sind die Ergebnisse des am 17. Juni 2011 vorgelegten Evaluierungsberichts der Bundestagsverwaltung über die Anwendung der Begleitgesetze zum Vertrag von Lissabon $^{5}$ in die Neufassung des EUZBBG eingeflossen. ${ }^{6}$

Im Hinblick auf die Unterrichtung des Bundestages in EU-Angelegenheiten verfolgt das neu gefasste Gesetz zwei Ziele, die in einem gewissen Spannungsverhältnis zueinander stehen. Einerseits geht es darum, den Unterrichtungsanspruch des Bundestages so allgemein und umfassend $\mathrm{zu}$ formulieren, dass die Fülle der gegenwärtigen und zukünftigen politischen Entwicklungen in Brüssel, Luxemburg und Straßburg erfasst wird. Dazu wurden der Begriff der EU-Angelegenheiten weit gefasst (siehe unter 1.) und für die gesamte Unterrichtung geltende Grundsätze aufgestellt (2.). Andererseits muss das EUZBBG im Interesse einer reibungslosen Zusammenarbeit auf der Arbeitsebene klarstellen, welche einzelnen Dokumente konkret von der Bundesregierung an den Bundestag zu übermitteln sind. Dies konnte erreicht werden durch eine noch konkretere Ausgestaltung der einschlägigen Detailregelungen (3.). Zusätzliche Unterrichtungspflichten knüpft das EUZBBG - wie bisher - an den Vorhabenbegriff, der allerdings seine Rolle als Ausgangspunkt der Unterrichtungsrechte des Bundestages in EU-Angelegenheiten eingebüßt hat (4.).

Ein weiteres Anliegen des novellierten Gesetzes war es, das Verhältnis der Unterrichtungsrechte aus dem EUZBBG zu den entsprechenden Bestimmungen des Stabilisierungsmechanismusgesetzes $\left(\right.$ StabMechG) 7 und des ESM-Finanzierungsgesetzes (ESMFinG) ${ }^{8}$ zu regeln (5.). Außerdem wurde im Zuge der Novellierung des Gesetzes auch das Stellungnahmeverfahren modifiziert (6.).

\section{Der Begriff der Angelegenheiten der Europäischen Union}

Das novellierte EUZBBG übernimmt in seiner Definition des Begriffs der Angelegenheiten der Europäischen Union in $\$ 1$ Abs. 2 die Formulierung des Bundesverfassungsgerichts vom 19. Juni 2012. EU-Angelegenheiten sind demnach nicht nur die Prozesse im Rahmen der Gemeinschaftsmethode, sondern auch Vorhaben auf völkerrechtlicher Grundlage außerhalb der Gründungsverträge, „wenn sie in einem Ergänzungs- oder sonstigen besonderen Nähe-

4 Vgl. BT-Drs. 17/12816, S. 8.

5 Vgl. BT-Drs. 17/14601.

6 Vgl. BT- Drs. 17/12816, S. 8.

7 Gesetz zur Übernahme von Gewährleistungen im Rahmen eines europäischen Stabilisierungsmechanismus vom 22. Mai 2010, BGBl. I, S. 627, das zuletzt durch Artikel 1 des Gesetzes vom 23. Mai 2012 (BGBl. I, S. 1166) geändert worden ist.

8 Gesetz zur finanziellen Beteiligung am Europäischen Stabilitätsmechanismus vom 13. September 2012, BGBl. I, S. 1918. 
verhältnis zum Recht der Europäischen Union stehen“9. Das EUZBBG definiert den Begriff - ebenso wie das BVerfG - nicht abschließend ${ }^{10}$ und trägt auf diese Weise dem Umstand Rechnung, dass die europäische Integration ein dynamischer Prozess ist, der Begriff der EUAngelegenheiten mithin ständiger Veränderung unterworfen ist.

\section{Die Grundsätze der Unterrichtung}

Den einzelnen Unterrichtungsrechten werden in $\$ 3$ EUZBBG die in jedem Fall geltenden Grundsätze der Unterrichtung vorangestellt. Demnach ist über alle EU-Angelegenheiten frühestmöglich, umfassend, fortlaufend und grundsätzlich schriftlich zu unterrichten. ${ }^{11}$ Diese Bestimmung ist zugleich eine Generalklausel, nach der auch dann zu unterrichten ist, wenn Dokumenttypen oder Verhandlungsformate im Wortlaut des Gesetzes nicht explizit erwähnt sind. Damit ist nicht mehr - wie bisher - der Vorhabenbegriff Ausgangspunkt für die Unterrichtungsrechte des Bundestages. Zwar war und ist auch dieser flexibel (,insbesondere“), in der Praxis gab es jedoch zum Teil unterschiedliche Auffassungen, inwieweit Dokumente, die keinem der in dem Katalog des bisherigen $₫ 3$ EUZBBG a.F. aufgezählten Vorhaben zuzuordnen waren, von den Unterrichtungsrechten des Bundestages erfasst waren. Dies galt vor allem für die Maßnahmen zur Stabilisierung des Euro auf Grundlage der völkerrechtlichen Verträge über den ESM und die EFSF. 12

Adressat der Unterrichtung ist grundsätzlich der Bundestag als Ganzer. Durch die Unterrichtung einzelner Abgeordneter oder Ausschüsse erfüllt die Bundesregierung ihre Unterrichtungspflichten aus dem EUZBBG nicht. Damit soll gewährleistet werden, dass sämtliche Abgeordnete gleichermaßen und unterschiedslos auf die übermittelten Informationen zugreifen können. ${ }^{13}$ Daher übersendet die Bundesregierung alle Dokumente stets auch an die Bundestagsverwaltung, von wo aus sie allen Abgeordneten in den Datenbanken des Bundestages zugänglich gemacht werden.

Die Unterrichtungspflicht umfasst alle Stadien der Entwicklung einer EU-Angelegenheit. $\$ 3$ Abs. 2 Satz 1 EUZBBG benennt dazu beispielhaft, aber nicht abschließend, Bereiche, auf die sich die Unterrichtung durch die Bundesregierung „insbesondere“ erstreckt. Besondere Bedeutung hat in diesem Zusammenhang die „Willensbildung der Bundesregierung“, da die Mitwirkung hieran letztlich das Ziel der im EUZBBG geregelten Beteiligungsrechte ist. ${ }^{14}$ Folglich leitet die Bundesregierung dem Bundestag Initiativen und Positionen zu, die sie für die EU-Ebene erstellt. ${ }^{15}$ Gleiches gilt für entsprechende Initiativen, Stellungnahmen,

9 BVerfGE 131, S. 152, S. 152 (1. Leitsatz).

10 Vgl. BT-Drs. 17/12816, S. 8.

11 Für eine allgemeine Definition dieser Begriffe siehe Wolff Heintschel von Heinegg, in: Volker Epping / Christian Hillgruber (Hrsg.), Beck'scher Online-Kommentar GG, Stand: 15. Mai 2013, Art. 23, Rn. 34.

12 Der Wortlaut der entsprechenden Begleitgesetze lässt daher offen, ob ESM und EFSF Angelegenheiten der EU sind.

13 Vgl. BVerfGE 131, S. 152, S. 213 f.

14 Vgl. \$1 Abs. 1 EUZBBG; BVerfGE 131, S. 152, S. 202 f.; BT-Drs. 17/12816, S. 9; Rupert Scholz, in: Theodor Maunz / Günter Dürig (Hrsg.), Grundgesetz. Kommentar, Loseblattsammlung, 69. Ergänzungslieferung, München 2013, Art. 23, Rn. 156.

15 Vgl. $\$ 4$ Abs. 2 Nr. 1 EUZBBG. 
Konsultationsbeiträge und Erläuterungen der übrigen Mitgliedstaaten ${ }^{16}$, des Bundesrates und der Länder. ${ }^{17}$ Weiterhin berücksichtigt das EUZBBG durchgehend, dass die Beratungen nicht nur im Rahmen der Sitzungen der EU-Organe selbst ${ }^{18}$, sondern auch in den diese vorbereitenden Gremien und Arbeitsgruppen stattfinden. ${ }^{19}$ Daher sind auch hierzu Berichte der Bundesregierung beziehungsweise der Ständigen Vertretung zu übermitteln. Überdies übersendet die Bundesregierung die Sammelweisungen für den deutschen Vertreter im Ausschuss der Ständigen Vertreter. ${ }^{20}$ Außerdem werden die im Rahmen der Sitzungen entstehenden Dokumente weitergeleitet. ${ }^{21}$

Die Anforderungen an den Zeitpunkt der Unterrichtung werden im novellierten EUZBBG präziser gefasst. ${ }^{22}$ Das Gebot der frühzeitigen Unterrichtung ergibt sich bereits aus Art. 23 Abs. 2 Satz 2 GG. Das neue EUZBBG verlangt, dass die Unterrichtung des Bundestages so rechtzeitig und dabei gleichzeitig so ausführlich erfolgt, dass er in die Lage versetzt wird, zu allen EU-Angelegenheiten auf einer soliden Informationsgrundlage eine Stellungnahme abzugeben. Dazu muss der Bundestag Informationen der Bundesregierung spätestens zu einem Zeitpunkt erhalten, der ihn in die Lage versetzt, sich fundiert mit dem Vorgang zu befassen und eine Position zu erarbeiten, bevor die Bundesregierung nach außen wirksame Erklärungen, insbesondere bindende Erklärungen zu EU-Rechtsetzungsakten und intergouvernementalen Vereinbarungen, abgibt. ${ }^{23}$ Konkret heißt dies, dass die Bundesregierung ihr vorliegende Dokumente zu EU-Angelegenheiten unverzüglich an den Bundestag weiterzuleiten hat. Ein Ermessen der Bundesregierung hinsichtlich des Zeitpunktes der Weiterleitung besteht nicht. Gibt es zu einem politischen Vorgang noch kein entsprechendes übermittlungsfähiges Dokument, hat die Bundesregierung selbst Berichte zu verfassen. Verzögerungen bei der Weiterleitung sind nur zulässig, um der Bundesregierung eine Prüfung der Voraussetzungen des Art. 23 Abs. 2 Satz 2 GG zu ermöglichen. ${ }^{24}$

Was die Qualität der Unterrichtung betrifft, verlangt das BVerfG, dass der Bundestag die für eine fundierte Beschlussfassung erforderlichen Informationen erhalten muss. Gleichzeitig hängt die Informationsdichte vom jeweiligen Sach- und Verhandlungsstand ab. 25

Der Grundsatz der fortlaufenden Unterrichtung wird in $\$ 4$ Abs. 1 Satz 2 EUZBBG konkretisiert. Bei der Unterrichtung ist die Entwicklung des Sach- und Verhandlungsstandes im Beratungsverlauf abzubilden. 26

Schließlich wird der Grundsatz der schriftlichen Unterrichtung besonders hervorgehoben. ${ }^{27}$ Der mündlichen Unterrichtung kommt demnach lediglich eine ergänzende und erläuternde Funktion zu. Ausnahmen vom Schriftlichkeitsgrundsatz sind nur zulässig und unter Umständen auch geboten, wenn eine frühestmögliche und umfassende Unterrichtung

16 Vgl. $\$ 4$ Abs. 2 Nr. 2 EUZBBG.

17 Vgl. $\$ 4$ Abs. 2 Nr. 3 EUZBBG.

18 Vgl. $\$ 3$ Abs. 2 ; $\$ 4$ Abs. 1 Nr. 1 lit a) und c), Abs. 1 Nr. 2, Abs. 4 EUZBBG.

19 Vgl. $\$ 3$ Abs. 2 Satz 2, Abs. 3 Satz 2; $\$ 4$ Abs. 1 Nr. 1 lit. c) EUZBBG.

20 Vgl. $\$ 4$ Abs. 2 Nr. 4 EUZBBG.

21 Vgl. $\$ 4$ Abs. 1 Nr. 1 lit. c) EUZBBG.

22 Vgl. insbesondere $₫ 3$ Abs. 1 Satz $4 ; \$ 4$ Abs. 1 Satz 2 EUZBBG.

23 Vgl. BVerfGE 131, S. 152, S. 152 f. (3. Leitsatz).

24 Vgl. ebenda, S. $212 \mathrm{f}$.

25 Vgl. ebenda, S. 206 f., S. 208; BT- Drs. 17/12816, S. 9.

26 Vgl. BVerfGE 131, S. 152, S. 152 (2. Leitsatz).

27 Vgl. $\$ 3$ Abs. 1 Satz 3 EUZBBG. 
- insbesondere in Eilfällen - anders nicht gewährleistet werden kann. ${ }^{28}$ Im Zusammenhang mit der schriftlichen Unterrichtung enthält $\$ 3$ Abs. 1 Satz 2 EUZBBG einen kurzen, aber wichtigen Hinweis darauf, dass sich die Unterrichtung nicht in der Weiterleitung von ohnehin bei der Bundesregierung vorhandenen Dokumenten erschöpft. Vielmehr ist diese verpflichtet, eigens für den Bundestag Berichte zu erstellen, wenn ihn wichtige Informationen zu einer EU-Angelegenheit ansonsten nicht erreichen würden.

Mit $\$ 3$ Abs. 4 EUZBBG wird der sich aus dem Grundgesetz ergebende Kernbereich exekutiver Eigenverantwortung als Grenze der Unterrichtungspflichten der Bundesregierung eingeführt. ${ }^{29}$ Solange ihre interne Willensbildung nicht abgeschlossen ist, besteht kein Anspruch des Parlaments auf Unterrichtung. 30 Wenn die Bundesregierung indes ihre Willensbildung selbst so weit konkretisiert hat, dass sie Zwischen- oder Teilergebnisse an die Öffentlichkeit geben kann oder mit einer eigenen Position in einen Abstimmungsprozess mit Dritten eintreten will, kann sie nicht mehr mit Verweis auf den Kernbereich exekutiver Eigenverantwortung eine Unterrichtung des Bundestages verweigern. ${ }^{31}$ Das bedeutet unter anderem, dass das Parlament zu unterrichten ist, wenn Mitglieder der Bundesregierung mit innerhalb der Ressorts noch nicht abgestimmten Initiativen zur EU-Politik an Dritte, insbesondere die EU-Organe oder Regierungen anderer Mitgliedstaaten, herantreten. ${ }^{32}$

$\$ 3$ Abs. 5 EUZBBG eröffnet - wie bisher - dem Bundestag die Möglichkeit, auf einzelne Unterrichtungen zu verzichten, es sei denn, eine Fraktion oder fünf von Hundert der Mitglieder des Bundestages widersprechen. Diese Bestimmung soll helfen, den Bundestag vor einer „Informationsüberflutung“ zu schützen ${ }^{33}$, hat bislang jedoch kaum praktische Relevanz erlangt.

\section{Konkretisierung der Unterrichtung}

$\$ 4$ EUZBBG konkretisiert die in $\$ 3$ EUZBBG geregelten Grundsätze, indem die einzelnen von der Bundesregierung zu übermittelnden Dokumententypen aufgeführt werden. Anders als $\$ \$ 4$ und 5 EUZBBG a.F. gilt diese Übermittlungspflicht unabhängig davon, ob ein Dokument sich einem Vorhaben im Sinne des $₫ 5$ EUZBBG zuordnen lässt.

Erfasst werden sollen alle bei der Bundesregierung vorliegenden Dokumente zu EUAngelegenheiten. Dazu gehören - wie bisher - die Dokumente der EU-Organe und Institutionen ${ }^{34}$, Dokumente, die die Bundesregierung für die EU-Ebene erstellt sowie der Bundesregierung vorliegende Dokumente anderer Mitgliedstaaten und des Bundesrates/der Länder für die EU-Organe. Außerdem hat die Bundesregierung ihre Berichte über die Verhandlungen auf EU-Ebene an den Bundestag zu übermitteln.

Die Unterrichtungspflicht erstreckt sich auch auf die vorbereitenden Gremien und Arbeitsgruppen. Über diese Verhandlungsformate hat die Bundesregierung zu berichten und

28 Vgl. BVerfGE 131, S. 152, S. 214 f.; BT-Drs. 17/12816, S. 9.

29 Vgl. zum Umfang des Kernbereichs BVerfGE 67, S. 100, S. 139.

30 Vgl. BVerfGE 131, S. 152, S. 206.

31 Vgl. ebenda, S. 210.

32 Vgl. BVerfGE 124, S. 78, S. 140.

33 Vgl. BVerfGE 131, S. 152, S. 208 f.; BT-Drs. 17/12816, S. 9.

34 Vgl. $\$ 4$ Abs. 1 Nummer 1; die bisherige allgemeine Zuleitung (vgl. $\$ 6$ Abs. 2 EUZBBG in der Fassung vom 13. September 2012) konnte daher entfallen. 
die dort entstehenden Dokumente an den Bundestag weiterzuleiten. Allerdings entstehen hier eine Vielzahl von Tischvorlagen und andere inoffizielle Dokumente, die unter Umständen schon nach kurzer Zeit nicht mehr aktuell sind. Für diese Dokumente kommt $\$ 4$ Abs. 3 EUZBBG zur Anwendung. ${ }^{35}$

In dieser Bestimmung wurde der Anspruch auf die Übermittlung inoffizieller Dokumente neu gefasst. Über diese ist zu informieren, sobald sie - gegebenenfalls auch über die Ständige Vertretung der Bundesrepublik Deutschland bei der Europäischen Union - in den Einflussbereich der Bundesregierung gelangen. ${ }^{36}$ Dies kann durch eine Auflistung der der Bundesregierung vorliegenden inoffiziellen Dokumente im Rahmen der Gremienberichterstattung (Drahtberichte, Vor- und Nachberichte etc.) erfolgen. ${ }^{37}$ Diese sind nur auf Anforderung zu übersenden, um eine Überflutung des Bundestages mit häufig nicht relevanten Dokumenten zu vermeiden. ${ }^{38}$ Anforderungsberechtigt sind einzelne Abgeordnete, die Ausschüsse, die Fraktionen und die Arbeitsebene. ${ }^{39}$ Die Bundesregierung muss inoffizielle Dokumente allerdings eigeninitiativ übermitteln, wenn eine frühestmögliche und umfassende Unterrichtung ansonsten nicht gewährleistet wäre. ${ }^{40}$ Dies wird insbesondere dann der Fall sein, wenn es zu neuen Initiativen oder Entwicklungen auf EU-Ebene noch keine offiziellen Dokumente gibt und entsprechende Planungen zunächst nur in inoffiziellen Papieren festgehalten sind.

Ausgeweitet wurde mit der Novellierung des EUZBBG vor allem die Unterrichtung über völkerrechtliche Verträge zwischen EU-Mitgliedstaaten, sofern es sich dabei um EU-Angelegenheiten handelt. Nicht nur über die Entstehung dieser Verträge ist zu informieren, sondern auch über den Verlauf der Beratungen in den Gremien, die auf der Grundlage zwischenstaatlicher Übereinkünfte zusammentreten sowie allgemein über die intergouvernementale Zusammenarbeit der Mitgliedstaaten. Dies betrifft unter anderem die Eurogruppe und den Eurogipfel. 41

$\$ 4$ Abs. 5 EUZBBG ergänzt die frühzeitige Unterrichtung durch die Verpflichtung der Bundesregierung, dem Bundestag mindestens vierteljährlich Frühwarnberichte über aktuelle politische Entwicklungen in Angelegenheiten der Europäischen Union zu übersenden. Diese regelmäßige Frühwarnberichterstattung ist kein Ersatz für eine frühestmögliche Unterrichtung im Einzelfall.

Ferner wird die Unterrichtung über Vertragsverletzungsverfahren konkretisiert und ausgeweitet. Dem Bundestag sind nicht mehr nur die Mahnschreiben und mit Gründen versehenen Stellungnahmen der Kommission, sondern auch erläuternde Informationen und Dokumente, insbesondere die Antwortschreiben der Bundesregierung, zuzuleiten. Weiterhin wird klargestellt, dass die Unterrichtungspflicht auch für Vertragsverletzungsverfahren gilt, die aufgrund einer unvollständigen oder fehlerhaften Umsetzung von Richtlinien eingeleitet werden. ${ }^{42}$ Die Unterrichtung über die Verfahren vor dem Europäischen Gerichtshof er-

35 Vgl. BT-Drs. 17/12816, S. 10.

36 Vgl. BVerfGE 131, S. 152, S. 207 f., S. 212 f., S. 220 f.

37 Vgl. BT-Drs. 17/12816, S. 10.

38 Vgl. BVerfGE 131, S. 152, S. 208 f.

39 Vgl. BT-Drs. 16/13925, S. 7.

40 Vgl. BT-Drs. 17/12816, S. 10.

41 Vgl. $₫ 3$ Abs. 3; $₫ 4$ Abs. 1 Nr. 1 lit. b), Abs. 1 Nr. 2 lt. a), Abs. 2 Nr. 1, Abs. 4; $\$ 5$ Abs. 1 Nr. 11 und 12 EUZBBG.

42 Vgl. \$4 Abs. 6 Nr. 1 EUZBBG. 
streckt sich schließlich auf Verfahren, bei denen die Bundesrepublik Verfahrensbeteiligte ist oder sich - zum Beispiel durch Stellungnahmen - beteiligt. ${ }^{43}$ Auf Anforderung übermittelt die Bundesregierung auch ihr vorliegende Dokumente zu weiteren Verfahren. ${ }^{44}$

\section{Unterrichtung über Vorhaben}

$\$ 5$ EUZBBG übernimmt den bisherigen Vorhabenkatalog inhaltlich unverändert, jedoch in gestraffter Form. Dazu zählen insbesondere alle legislativen Vorschläge wie Verordnungen und Richtlinien, nicht-legislative Dokumente wie Mitteilungen, Grünbücher, Aktionspläne und Berichte, außerdem Verhandlungsmandate für völkerrechtliche Verträge der EU. Der Vorhabenkatalog war bereits durch das Änderungsgesetz vom 13. September 2012 erweitert worden. ${ }^{45}$ Demnach werden nunmehr auch Entwürfe völkerrechtlicher Verträge zwischen den Mitgliedstaaten, wenn sie in einem Ergänzungs- oder sonstigen besonderen Näheverhältnis zum Recht der Europäischen Union stehen, als Vorhaben behandelt. ${ }^{6}$ Gleiches gilt für Beratungsgegenstände, Vorschläge und Initiativen, die im Rahmen dieser Verträge behandelt werden. ${ }^{47}$ Der Vorhabenkatalog des $\$ 5$ Abs. 1 EUZBBG ist nach wie vor nicht abschließend („,insbesondere“). ${ }^{48}$

Obwohl die Unterrichtung nicht mehr an den Vorhabenbegriff geknüpft ist, hat dieser weiterhin eine wichtige Funktion für die Unterrichtungspraxis. So regelt $₫ 6$ EUZBBG speziell für Vorhaben, wie diese dem Bundestag zu übermitteln sind, nämlich im Wege der förmlichen Zuleitung. ${ }^{49}$ Außerdem gelten bestimmte Unterrichtungspflichten nur für Vorhaben. Dazu gehören - wie bisher - Berichtsbögen 50 und „Umfassende Bewertungen“ 51 .

Neu ist die in $\$ 6$ Abs. 5 EUZBBG normierte Pflicht der Bundesregierung zu besonders komplexen oder bedeutsamen Vorhaben auf Anforderung vertiefende Berichte zu erstellen. Damit wird der Aussage des Bundesverfassungsgerichts Rechnung getragen, wonach eine umso intensivere Unterrichtung geboten ist, je komplexer ein Vorgang ist, je tiefer er in den Zuständigkeitsbereich des Parlaments eingreift und je mehr er sich einer förmlichen Beschlussfassung oder Vereinbarung annähert. 52 Ein zusätzlicher Bericht soll nach der Intention des Gesetzgebers nur angefordert werden, wenn die Summe der Informationen der Bundesregierung zu einem Vorhaben als nicht ausreichend erachtet wird. 53 Wer konkret anforderungsberechtigt ist, geht - ebenso wie bei $\$ 4$ Abs. 3 EUZBBG - weder aus dem Gesetzeswortlaut noch aus der Begründung hervor. Diese Parallele zur Anforderung inoffizieller Dokumente spricht allerdings dafür, dass sowohl aus dem politischen Raum als auch von der Arbeitsebene vertiefende Berichte bestellt werden können.

43 Vgl. $\$ 4$ Abs. 6 Nr. 2 EUZBBG.

44 Vgl. $\$ 4$ Abs. 6 Nr. 3 EUZBBG.

45 Vgl. BGBl. 2012 II, S. 1006.

46 Vgl. $\$ 5$ Abs. 1 Nr. 11 EUZBBG.

47 Vgl. $\$ 5$ Abs. 1 Nr. 12 EUZBBG.

48 Vgl. BT-Drs. 17/12816, S. 10.

49 Vgl. $\$ 6$ Abs. 1 EUZBBG.

50 Vgl. $\$ 6$ Abs. 2 EUZBBG.

$51 \$ 6$ Abs. 3 EUZBBG.

52 Vgl. BVerfGE 131, S. 152, S. 206 f.

53 Vgl. BT-Drs. 17/12816, S. 11. 


\section{Sonderregeln für Dokumente aus den Bereichen GASP/GSVP sowie ESM und EFSF}

Nach der Rechtsprechung des Bundesverfassungsgerichts handelt es sich beim Europäischen Stabilitätsmechanismus (ESM) und bei der Europäischen Finanzstabilisierungsfazilität (EFSF) um Angelegenheiten der Europäischen Union. ${ }^{54}$ Demnach kommt für die Unterrichtung grundsätzlich das EUZBBG zur Anwendung. Allerdings gibt es für diese beiden Instrumente mit dem ESMFinG und dem StabMechG bereits zwei Begleitgesetze mit eigenen Unterrichtungsrechten. Daher war es bei der Novellierung des EUZBBG erforderlich, das Verhältnis der Unterrichtungsrechte aus beiden Regelungskomplexen - EUZBBG auf der einen, ESMFinG und StabMechG auf der anderen Seite - zu klären. Dies konnte durch $\$ 5$ Abs. 3 EUZBBG erreicht werden, wonach für die Unterrichtung über Angelegenheiten des ESM beziehungsweise der EFSF zunächst die für alle EU-Angelegenheiten geltenden Bestimmungen der $\$ \$ 1$ bis 4 EUZBBG gelten, also insbesondere die in $₫ 3$ EUZBBG verankerten Grundsätze der Unterrichtung und die in $\$ 4$ EUZBBG konkretisierte Pflicht zur Übersendung aller der Bundesregierung vorliegenden Dokumente in diesem Bereich. Angelegenheiten des ESM beziehungsweise der EFSF unterfallen allerdings nicht dem Vorhabenbegriff. 55 Anstelle der vorhabenspezifischen Unterrichtungen, die in $\$ 6$ EUZBBG verankert sind, kommen die speziell für den ESM beziehungsweise die EFSF geltenden Unterrichtungsrechte aus $₫ 7$ ESMFinG beziehungsweise $\$ 5$ StabMechG zur Anwendung.

Maßnahmen im Bereich der Gemeinsamen Außen- und Sicherheitspolitik (GASP)/Gemeinsamen Sicherheits- und Verteidigungspolitik (GSVP) sind - wie bisher - keine Vorhaben. 56 Bundestagsintern werden aber die gemäß $\$ 7$ Abs. 1 EUZBBG von der Bundesregierung übermittelten Übersichten der Rechtsakte, deren Beratung absehbar bevorsteht, wie Vorhaben behandelt. 57

\section{Das Stellungnahmeverfahren}

Die Bestimmungen über das Verfahren zur Abgabe von Stellungnahmen des Bundestages zu EU-Angelegenheiten bleiben im Wesentlichen unverändert. Allerdings fasst $₫ 8$ Abs. 1 Satz 2 EUZBBG die Pflicht, die zur Bestimmung des Zeitpunkts zur Abgabe einer Stellungnahme erforderlichen Informationen zu übermitteln, konkreter; nunmehr muss der Bundestag fortlaufend über den Beratungsablauf informiert werden, so dass es ihm ermöglicht wird, den optimalen Zeitpunkt für eine Stellungnahme zu bestimmen. ${ }^{58}$

Ebenfalls modifiziert wurde die Pflicht der Bundesregierung, einen Parlamentsvorbehalt einzulegen. Weicht die Bundesregierung von einer Stellungnahme des Bundestages ab, hat sie nunmehr nicht erst auf der Ratstagung 59 , sondern bereits in dessen Vorbereitungsgremien einen solchen Parlamentsvorbehalt einzulegen. Dies gilt auch für Gremien und Institutionen auf völkerrechtlicher beziehungsweise intergouvernementaler Grundlage. ${ }^{60}$ Damit

54 Vgl. BVerfGE 131, S. 152, S. 152 (1. Leitsatz), S. 221 f.

55 Vgl. $\$ 5$ Abs. 3 Nr. 2 und 3 EUZBBG.

56 Vgl. $\$ 5$ Abs. 3 Nr. 1 EUZBBG.

57 Vgl. $\$ 93$ Abs. 3 Satz 1 GO-BT.

58 Vgl. BT-Drs. 17/12816, S. 11.

59 Vgl. \$9 Abs. 4 Satz 1 EUZBBG a.F.

60 Vgl. BT-Drs. 17/12816, S. 11. 
wird das Verfahren an die tatsächlichen Entscheidungsprozesse auf EU-Ebene angepasst. Wichtige Entscheidungen zeichnen sich in vielen Fällen bereits in den Vorbereitungsgremien des Rates ab. Um dem Rechnung zu tragen, wurde auf die Nennung konkreter Formate und Gremien zur Einlegung eines Parlamentsvorbehalts verzichtet. ${ }^{61}$

Neu eingefügt ist außerdem die Regel, dass auf Verlangen eines Viertels der Mitglieder des Bundestages die Bundesregierung im Rahmen einer Plenardebatte erläutern muss, warum sie von einer Stellungnahme des Bundestages abweichen will.62

Die bereits bisher vor der Aufnahme von Verhandlungen über Beitritte zur EU und für Vertragsänderungen geltenden besonderen Regelungen, wonach die Bundesregierung gemäß $\$ 9$ Abs. 1 EUZBBG auf das Stellungnahmerecht gesondert hinweist und gemäß $₫ 9$ Abs. 2 EUZBBG ein Einvernehmen herstellen soll (bei sonstigen Stellungnahmen ist ein „Bemühen“ ausreichend), werden nach $\$ 9$ a EUZBBG auf Entscheidungen des Rates nach Art. 140 Abs. 2 AEUV ausgedehnt. Durch einen solchen Beschluss wird die Einführung des Euro in einem weiteren Mitgliedstaat ermöglicht. Entsprechend werden Vorschläge und Initiativen für Beschlüsse gemäß Art. 140 Abs. 2 AEUV in den Vorhabenkatalog des $\$ 5$ Abs. 1 EUZBBG aufgenommen.

\section{Weitere Regelungen des neuen EUZBBG}

Die Bestimmung des $\$ 2$ EUZBBG zum Ausschuss für die Angelegenheiten der Europäischen Union wurde an den Wortlaut des Art. 45 GG angepasst. Die Bestimmung hat über die Wiederholung des Wortlauts des Grundgesetzes hinaus keinen eigenständigen Regelungsgehalt. 63

$\$ 10$ EUZBBG, der den Zugang zu Datenbanken und die vertrauliche Behandlung von Dokumenten regelt, bleibt unverändert, weil bereits das EUZBBG a.F. dem Interesse an einer vertraulichen Behandlung von EU-Dokumenten ausreichend Rechnung getragen hatte. Das BVerfG hatte in einer Geheimhaltungspflicht keinen Hinderungsgrund gesehen, eine Information an den Bundestag weiterzugeben. ${ }^{64}$ Sofern erforderlich, könne die Unterrichtung vertraulich erfolgen. ${ }^{65}$ Die Voraussetzungen dafür hat der Bundestag mit dem Erlass seiner Geheimschutzordnung geschaffen. ${ }^{66}$

Die Regelungen über das Verbindungsbüro des Bundestages in Brüssel werden von der Vereinbarung zwischen dem Bundestag und der Bundesregierung über die Zusammenarbeit in Angelegenheiten der Europäischen Union (BBV) 67 in $\$ 11$ EUZBBG überführt. Durch

61 Vgl. \$8 Abs. 2 EUZBBG; BT-Drs. 17/12816, S. 11.

62 Vgl. $\$ 8$ Abs. 5 Satz 2 EUZBBG.

63 Vgl. zur Sonderstellung des EU-Ausschusses Rupert Scholz, in: Theodor Maunz / Günter Dürig (Hrsg.), a.a.O. (Fn. 14), Art. 23, Rn. 155.

64 Vgl. BVerfGE 131, S. 152, S. 207.

65 Vgl. BVerfGE 124, S. 78, S. 123 f.

66 Vgl. BVerfGE 67, S. 100, S. 135; BVerfGE 70, S. 324, S. 359; BVerfGE 77, S. 1, S. 48; BVerfG 130, S. 318, S. 362.

67 Vereinbarung zwischen dem Deutschen Bundestag und der Bundesregierung über die Zusammenarbeit in Angelegenheiten der Europäischen Union in Ausführung des $\$ 6$ des Gesetzes über die Zusammenarbeit von Bundesregierung und Deutschem Bundestag in Angelegenheiten der Europäischen Union vom 28. September 2006, BGBl. I, S. 2177. 
die teilweise Neuformulierung sollen sich aber die Aufgaben des Verbindungsbüros nicht ändern. Da es sich um die letzte originäre Regelung der BBV gehandelt hat, kann diese aufgehoben werden. ${ }^{68}$

\title{
8. Ausblick: Wird sich das neue EUZBBG in der Praxis bewähren?
}

Insgesamt enthält das EUZBBG eine Reihe wesentlicher Verbesserungen gegenüber der bisher geltenden Fassung. Die weite und für neuere Entwicklungen offene Definition des Begriffs der Angelegenheiten der Europäischen Union und die Generalklausel in $₫ 3$ EUZBBG dürften sicherstellen, dass die Unterrichtung des Bundestages auch über künftige Integrationsschritte einfachgesetzlich gewährleistet ist. Die tägliche Zusammenarbeit zwischen Parlament und Regierung wird außerdem erleichtert durch die ausführlichen Bestimmungen zu den im Einzelnen zu übermittelnden Dokumenten. Außerdem gibt die Modifizierung des Stellungnahmeverfahrens dem Bundestag die Möglichkeit, dieses Recht künftig noch wirksamer als bisher wahrzunehmen.

68 Vgl. BT-Drs. 17/12816, S. 12.

\section{Der Unterausschuss Kommunales und die Verfahrensvorgaben der Geschäftsordnung des Deutschen Bundestages}

\author{
Heinz-Willi Heynckes
}

Im parlamentarischen Sprachgebrauch sind die Unterausschüsse des Deutschen Bundestages mit einer ebenso interessanten wie signifikanten Begriffsprägung verbunden: der „Atomisierung des Parlaments" 1 . Mit der Institutionalisierung von Unterausschüssen durch die große Geschäftsordnungsreform von $1980^{2}$ sollte dieser Gefahr und der nicht mehr überschaubaren Vielfalt von unterausschussähnlichen Einrichtungen im Bundestag - Kommissionen, Unterkommissionen, Arbeitsgruppen, Berichterstatter - begegnet werden. ${ }^{3}$ Auch der damalige Vorsitzende des Ausschusses für Wahlprüfung, Immunität und Geschäftsordnung4,

1 Der Begriff der Atomisierung des Parlaments fand sogar mediale Beachtung, vgl. Friedrich Karl Fromme, Zerfällt der Bundestag in seine Ausschüsse - Der Geschäftsordnungsausschuss sieht Anlass für eine Mahnung, in: FAZ vom 13. Dezember 1977.

2 Geschäftsordnung des Deutschen Bundestages in der Fassung der Bekanntmachung vom 2. Juli 1980, BT-Drs. 8/4127.

3 Manfred Schulte, MdB, 8. Wahlperiode, Stenographisches Protokoll 225. Sitzung am 25. Juni 1980, S. 18269; BT-Drs. 8/3460, S. 91; BT-Drs. 8/4127, S. 38; Heinrich G. Ritzel / Joseph Bücker / Hermann J. Schreiner / Helmut Winkelmann, Handbuch für die parlamentarische Praxis mit Kommentar zur Geschäftsordnung des Deutschen Bundestages, 27. Lieferung, Neuwied 2010, $\$ 55$, Bem. Nr. I. 1. a); Hans Troßmannn / Hans-Achim Roll, Parlamentsrecht, Ergänzungsband, München 1981, $\$ 55$, Rz. 3.

4 Im folgenden: GO-Ausschuss. 